\title{
Effects of sheath instability on properties of a Hall thruster
}

\author{
D. Sydorenko* \\ University of Saskatchewan, Saskatoon, SK, S7N5E2, Canada \\ I. D. Kaganovich ${ }^{\dagger}$ and Y. Raitses ${ }^{\star}$ \\ Plasma Physics Laboratory, Princeton University, Princeton, NJ, 08543, USA \\ and \\ A. Smolyakov ${ }^{\S}$ \\ University of Saskatchewan, Saskatoon, SK, S7N5E2, Canada
}

\begin{abstract}
Recent analytical studies and particle-in-cell simulations suggested that the electron velocity distribution function in a Hall thruster plasma is non-Maxwellian and anisotropic. The electron average kinetic energy in the direction parallel to the walls of a thruster is several times larger than the electron average kinetic energy in the direction normal to the walls. Electrons are stratified into several groups depending on their origin and confinement. These kinetic effects lead to many new properties of plasma in a Hall thruster. In particular, the sheath near the electron-emitting surface may become unstable if it is characterized by the negative electron conductivity. In this paper, the negative conductivity effects in Hall thrusters are studied making use of a one-dimensional particle-in-cell code. In case of intense turbulent heating, a new regime with relaxation sheath oscillations is observed. In this regime, the plasma switches between a state with non-space charge limited emission and a state with space charge limited emission. Transition to the latter state occurs when the negative conductivity appears due to modification of the velocity distribution of the plasma bulk
\end{abstract}

\section{Nomenclature}

$\mathrm{x}=$ coordinate normal to the walls, the direction of the applied magnetic field

$\mathrm{z}=$ coordinate parallel to the walls, the direction of the applied electric field

$\mathrm{t} \quad=$ time

$\mathrm{v}_{x, y, z} \quad=$ velocity components of an electron

$\mathrm{W}=$ kinetic energy of an electron

$w_{x, y, z} \quad=$ kinetic energy of electron motion in the $x, y, z$ directions respectively

$\mathrm{m} \quad=$ electron mass

$\mathrm{M} \quad=$ ion mass

$\mathrm{e} \quad=$ elementary charge

$\mathrm{H} \quad=$ width of the plasma slab

$E_{x, z} \quad=$ components of the electric field (the self-consistent field is normal to the wall)

$B_{x} \quad=$ applied magnetic field

$\Phi=$ = electrostatic potential relative to the dielectric wall at $\mathrm{x}=\mathrm{H}$

$n_{a} \quad=$ neutral gas density

$n_{e} \quad=$ electron density

$v_{\text {turb }} \quad=$ frequency of "turbulent" collisions

$v_{e n} \quad=$ frequency of electron-neutral collisions

\footnotetext{
* At present time - postdoctoral fellow, University of Alberta, Edmonton, sydorenk@ualberta.ca.

${ }^{\dagger}$ Research physicist, Princeton Plasma Physics Laboratory, ikaganov@pppl.gov, AIAA member.

*esearch physicist, Princeton Plasma Physics Laboratory, yraitses@pppl.gov, AIAA member.

$\S$ Professor, University of Saskatchewan, Saskatoon, andrei.smolyakov@usask.ca, AIAA member.
} 


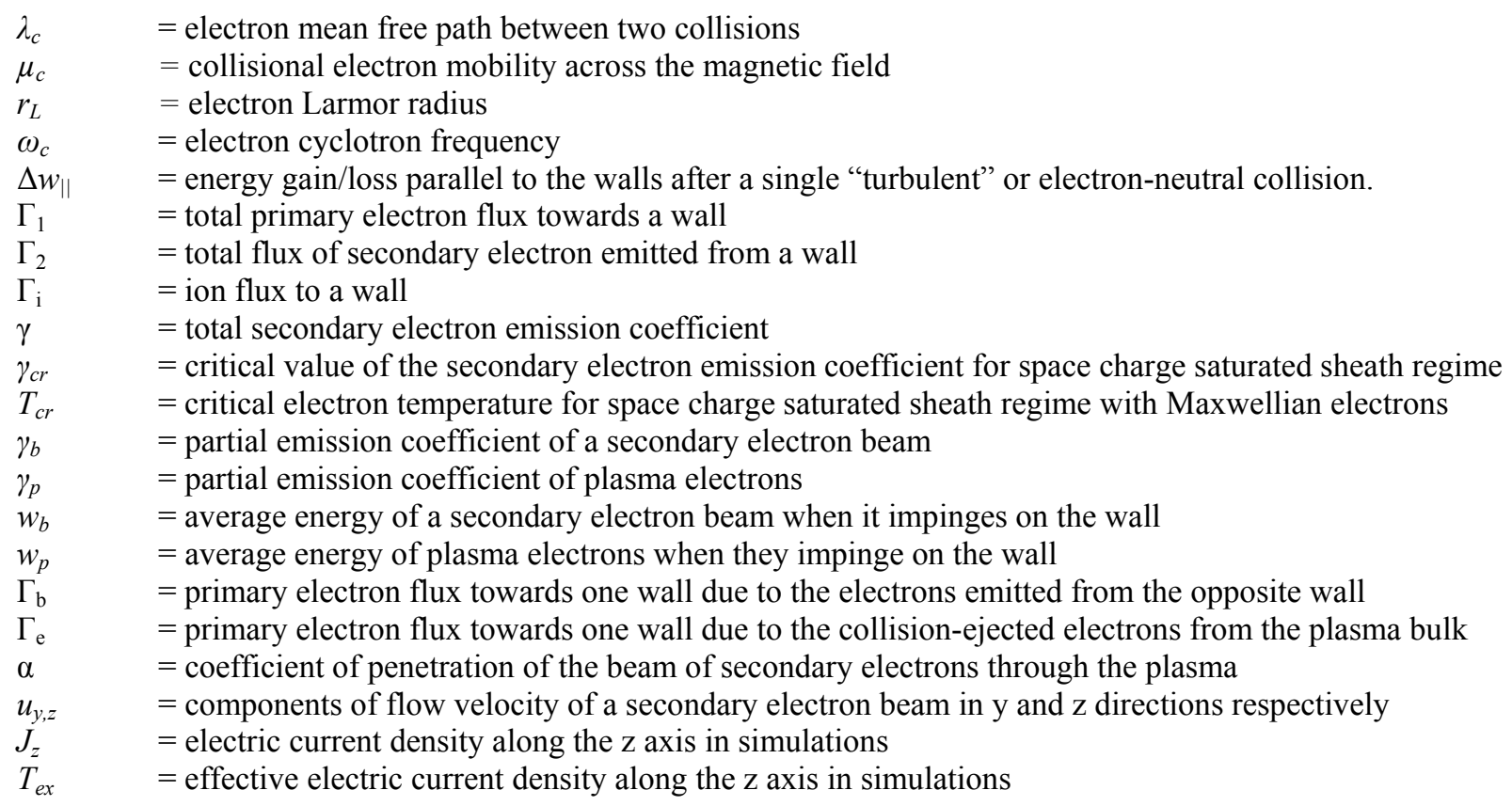

\section{Introduction}

$\mathrm{T}_{\mathrm{h}}^{\mathrm{h}}$ here is a reliable experimental evidence of the wall material effect on operation of a Hall thruster. ${ }^{1,2}$ The existing fluid theories explain this effect invoking a strong secondary electron emission (SEE) from the channel walls. The SEE is predicted to weaken insulating properties of the near-wall sheaths and, thereby, (i) to cause cooling of plasma electrons and (ii) to enhance the electron conductivity across the magnetic field. From a practical standpoint, a strong SEE from the channel walls is expected to cause additional inefficiencies due to enhanced power losses in the thruster discharge, and intense heating of the channel walls by almost thermal electron fluxes from the plasma ${ }^{3}$. Moreover, because the SEE may lead to lower values of the sheath potential drop, ion-induced erosion of the channel walls can be also affected. Although these predictions can be certainly applied for plasmas with a Maxwellian electron velocity distribution function (EVDF), there is no consensus between the existing fluid ${ }^{2,4,5,6,7}$ and kinetic models ${ }^{8,9}$ on how strong the SEE effects on the thruster plasma are. According to kinetic simulations ${ }^{10,11,12,13}$ the EVDF in a collisionless plasma is depleted at high-energies due to electron-wall losses. Under such conditions, the electron losses to the walls can be hundreds of times smaller than the losses predicted by the fluid theories. A similar depletion of EVDF at high energies was also reported for other kinds of low-pressure gas discharges. ${ }^{14,15,16}$ Note that the deviation of the EVDF from a Maxwellian does not necessarily mean that the SEE cannot play a significant role in the thruster discharge. In experiments with a Hall thruster operating at high discharge voltages, the maximum electron temperature and the electron cross-field current were strongly affected by the SEE properties of the channel wall materials, ${ }^{17,18}$.

In recent particle-in-cell (PIC) simulations ${ }^{10,11,12}$ and in the kinetic analytical study, ${ }^{14}$ we showed that the SEE effect on power losses in a thruster discharge is quite different from what was predicted by previous fluid and kinetic studies. In simulations, the EVDF was found to be strongly anisotropic, depleted at high energies, and in some cases, even non-monotonic. The average kinetic energy of electron motion in the direction parallel to the walls is several times larger than the average kinetic energy of electron motion in the direction normal to the walls. Secondary electrons form two beams propagating between the walls of a thruster channel in opposite radial directions ${ }^{10,11}$ (also predicted in Ref. 19 in the modified fluid approximation). In this paper, we highlight results of recent kinetic studies of HT (Refs. 10-14) which are summarized in the form of convenient analytical formulas for predicting kinetic properties of HT plasma. It is shown that for a typical high-performance Hall thruster, the electron fluxes to the walls are limited by the source of electrons, overcoming the wall potential and leaving the plasma. The flux of these electrons is determined mainly by the frequencies of elastic electron collisions with atoms and ions. The sheath insulating properties depend on the electron fluxes to the walls and, therefore, on the rate of elastic scattering of plasma electrons. 
In previous kinetic studies, Meezan and Cappelli ${ }^{8}$ developed a kinetic model based on the so-called nonlocal approach. The non-local approach (described for example in Ref. 20) was developed for large gas discharges with the distance between walls of order tens of centimeters and at pressures above $10 \mathrm{mTorr}$, where the electron mean free path is much smaller than the discharge gap $\lambda_{\mathrm{c}}<<\mathrm{H}$. In Hall thrusters the characteristic distance between walls is given by the channel width. Because of the smallness of the electron mean free path in these gas discharges, the EVDF is isotropic even for electrons with energy high enough to overcome the wall potential. However, the traditional nonlocal approach is not applicable to Hall thrusters, which operate in the opposite limit $\lambda_{\mathrm{c}}>>\mathrm{H}$. Because the electron mean free path in Hall thrusters is much larger than the channel width, the EVDF has been shown to be anisotropic ${ }^{11}$. Moreover, the anisotropy of the EVDF strongly affects the electron flux to the wall, as shown below. Practical analytical formulas are derived for wall fluxes, secondary electron fluxes, plasma parameters and contribution to the electron current due to SEE. The calculations based on the analytical formulas agree well with the results of numerical simulations.

An important implication of the present work is that future theoretical and experimental studies need to determine the influence of these kinetic effects on the thruster performance and heating and erosion of the channel walls. For instance, the reduction of the gas density in the thruster channel might significantly reduce the electron fluxes to the walls because in xenon plasmas of Hall thrusters the electron collisions with neutral atoms is the major scattering process while the Coulomb scattering off the ions gives a small contribution.

\section{Sheath Instability}

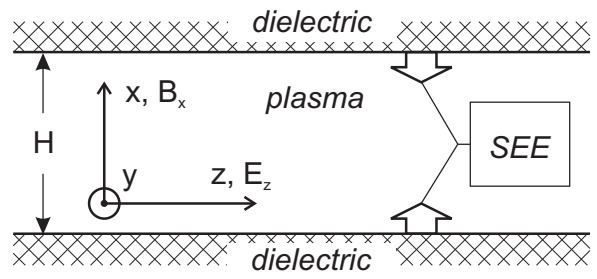

FIG. 1: Schematic diagram of the Hall thruster model. The two dielectric walls represent the coaxial ceramic channel of a Hall thruster.

The sheath near the electron-emitting surface may become unstable due to the strong departure of EVDF from a Maxwellian $\mathrm{EVDF}^{21}$. PIC simulation of Hall thruster plasmas in the plane geometry model shown schematically in Fig. 1 (the description of the code is given elsewhere ${ }^{23}$ ) reveals a plasma-sheath instability manifesting itself as a rearrangement of the plasma sheath near the thruster channel walls accompanied by a sudden change of many discharge parameters. The instability develops when the sheath current as a function of the sheath voltage is in the negative conductivity regime. The major part of the sheath current is produced by beams of secondary electrons counter-streaming between the walls. The negative conductivity is the result of nonlinear dependence of beam-induced secondary electron emission on the plasma potential. The intensity of such emission is defined by the beam energy. The energy of the beam in crossed axial electric and radial magnetic fields is a quasi-periodical function of the phase of cyclotron rotation, which depends on the radial profile of the potential and the thruster channel width. There is a discrete set of stability intervals determined by the final phase of the cyclotron rotation of secondary electrons. As a result, small variation of the thruster channel width may result in abrupt changes of plasma parameters when the plasma state jumps from one stability interval to another. In PIC simulations, we found that also the relaxation sheath oscillations (RSO) may appear.

A PIC simulation of a thruster-like system was carried out as follows. The constant simulation parameters were the distance between the walls $\mathrm{H}=2.5 \mathrm{~cm}$, the axial electric field $\mathrm{E}_{\mathrm{z}}=200 \mathrm{~V} / \mathrm{cm}$, the magnetic field $\mathrm{B}_{\mathrm{x}}=100$ Gauss, the neutral xenon atom density $n_{a}=10^{12} \mathrm{~cm}^{-3}$, and the turbulent collision frequency $v_{t}=2: 810^{6} \mathrm{~s}^{-1}$. Initially, the plasma (xenon) has uniform density $n_{0}=10^{11} \mathrm{~cm}^{-3}$ and isotropic electron temperature $T e x=T e y=T e z=10 \mathrm{eV}$. The electron component had a drift in the $y$-direction with the velocity $V_{d r}$. The simulation parameters were not chosen so as to reproduce some regime of a real Hall thruster, but rather to investigate the plasma-wall interaction and the regime of the sheath with SEE while under the condition of intense turbulent heating. In particular, the space-charge limited $(\mathrm{SCL}) \mathrm{SEE}$ regime ${ }^{13}$ with $\gamma>\gamma_{c r}$ (for xenon plasma $\gamma_{c r}=0: 983$ ) and a non-monotonic potential profile in the sheath was expected. In the simulation, the plasma became energetic and strongly anisotropic with the $53 \mathrm{eV}$ of the average energy of electron motion in the $\mathrm{y}$-direction, $42 \mathrm{eV}$ of that in the z-direction, and $4.2 \mathrm{eV}$ in the $\mathrm{x}$-direction. The SCL 

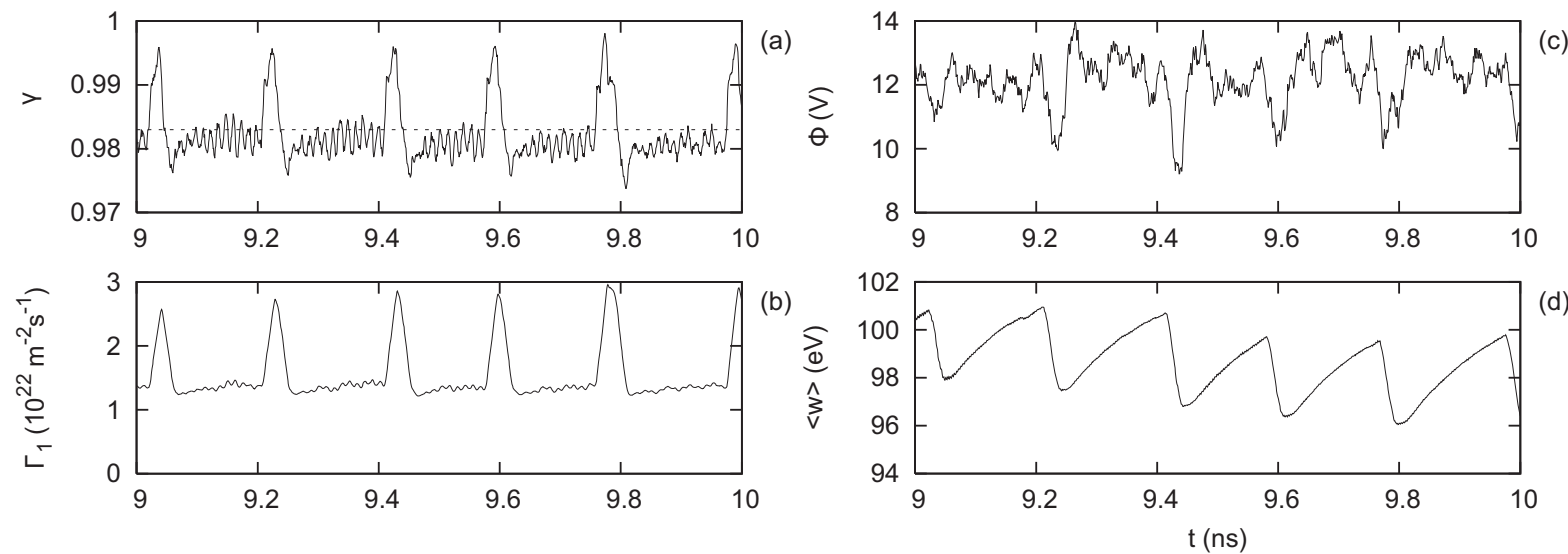

FIG. 2: Evolution of general plasma parameters with time in the RSO regime. (a) Total emission coefficient $\gamma$ at the wall $\mathrm{x}=\mathrm{H}$ (solid curve) and the threshold emission coefficient for the SCL SEE (dashed line). (b) Total primary electron flux $\Gamma_{1}$ to the wall $\mathrm{x}=\mathrm{H}$. (c) Electrostatic potential in the middle of the plasma $\Phi_{\mathrm{p}}$. (d) Average electron energy $\Re \sqsupseteq T \swarrow$

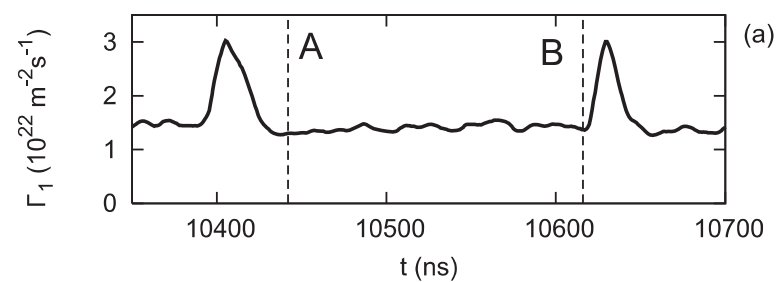

EVDF (rel.un.)

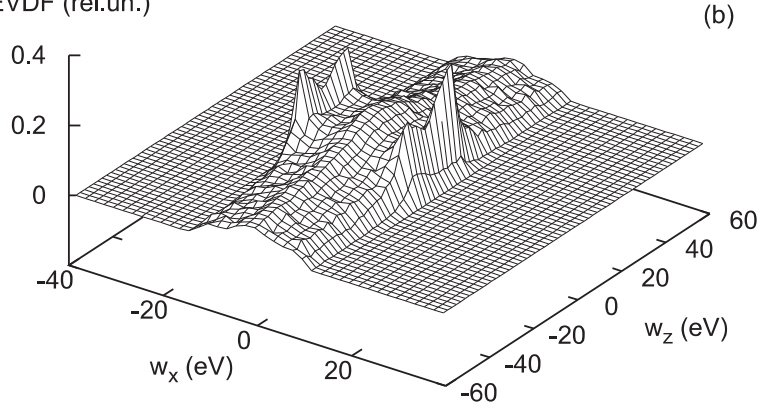

EVDF (rel.un.)
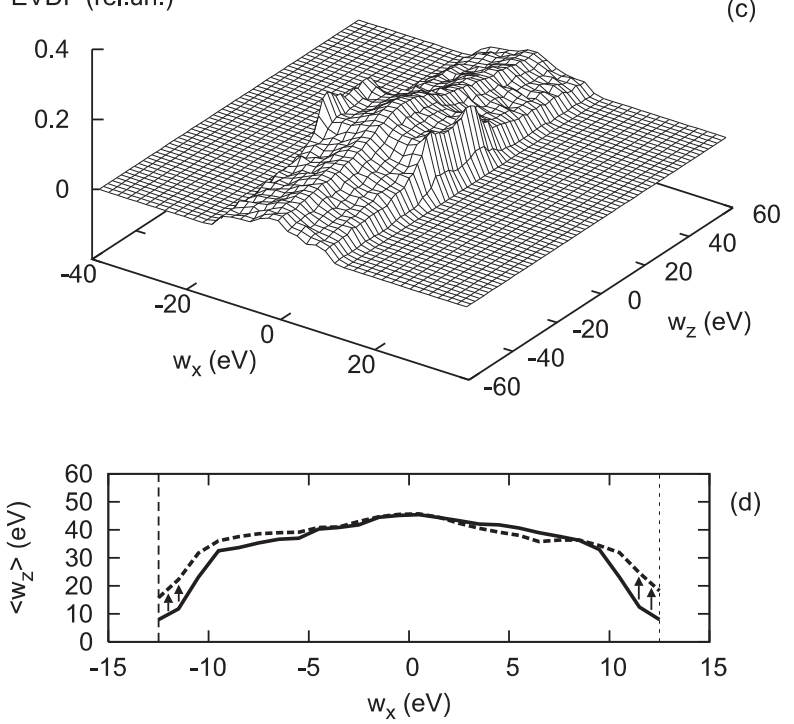

FIG. 3: (a) The primary electron flux to the wall $\mathrm{x}=\mathrm{H}$ during one RSO period, vertical lines A and $\mathrm{B}$ mark the beginning (time $t_{A}$ ) and the end (time $t_{B}$ ) of the non-SCL state. (b) and (c) The EVDF over $v_{x}$ and $v_{z}$ in the middle of the plasma $10 \mathrm{~mm}<\mathrm{x}<15 \mathrm{~mm}$ obtained at times $\mathrm{t}_{\mathrm{A}}$ (b) and $\mathrm{t}_{\mathrm{B}}(\mathrm{c})$. (d) The dependence at times $\mathrm{t}_{\mathrm{A}}$ (solid curve) and $\mathrm{t}_{\mathrm{B}}$ (dashed curve). Vertical lines in (d) mark the electron confinement threshold $\Phi_{\mathrm{p}}$. Graphs in (b), (c), and (d) are plotted in energy coordinates, negative energy values correspond to propagation in the negative direction.

SEE regime was observed in the simulation, but it never established as a stationary state. Instead, it was found that the system reaches a quasi-periodic regime, where it oscillates between the SCL and non-SCL states. The SCL state lasts for a short time, while the non-SCL state lasts much longer (the width of the spikes in Fig. 2a is much smaller than the intervals between the spikes). In the SCL state, the primary electron flux grows abruptly and is several times higher than during the non-SCL state (see Fig. 2b) and the plasma potential decreases (see Fig. 2c).

In Fig.3 (b) and (c), we represent the bulk plasma electron distribution function over the $\mathrm{x}$ and $\mathrm{z}$ velocities in the midplane, $\mathrm{f}\left(\mathrm{v}_{\mathrm{x}}, \mathrm{v}_{\mathrm{y}}, \mathrm{H} / 2\right)$, obtained during the non-SCL stage of one RSO period shown in Fig.3(a). At the time $t_{A}$ marked by vertical line A in Fig.3(a), which is immediately after the end of the SCL stage, the EVDF has highamplitude spikes created by numerous cold secondary electrons trapped by the restored plasma potential, see 
Fig.3(b). With time, these spikes disperse because electrons (i) suffer turbulent collisions (the anisotropic Joule heating), and (ii) are mixed in the phase space $\mathrm{x}-\mathrm{v}_{\mathrm{x}}$ by a weak two-stream instability. ${ }^{13}$ By the end of the non-SCL stage [time $t_{B}$ marked by vertical line B in Fig.3(a)] the amplitude of the spikes in the EVDF decreases by a factor of two, see Fig.3(c). To give a quantitative characteristic of the local EVDF modification, in Fig.3(d) we plotted dependences $<\mathrm{w}_{\mathrm{z}}\left(\mathrm{v}_{\mathrm{x}}\right)>$ for the plasma bulk electrons with $\mathrm{w}_{\mathrm{x}}<\mathrm{e} \Phi_{\mathrm{p}}$ at times $\mathrm{t}_{\mathrm{A}}$ and $\mathrm{t}_{\mathrm{B}}$, where

$$
\left\langle w_{z}\left(v_{x}\right)\right\rangle=\int_{-\infty}^{\infty} d v_{z} \frac{m v_{z}^{2}}{2} f\left(v_{x}, v_{z}, H / 2\right) \text {. }
$$

The dashed curve $<\mathrm{W}_{\mathrm{z}}\left(\mathrm{v}_{\mathrm{x}}\right)>$ obtained at time $\mathrm{t}_{\mathrm{B}}$ is clearly higher than the solid curve obtained at time $\mathrm{t}_{\mathrm{A}}$ in the region $10 \mathrm{eV}<\mathrm{w}_{\mathrm{x}}<12.5 \mathrm{eV}$. In other words, by the end of the non-SCL stage, the electrons with $\Phi_{\mathrm{p}}-\delta \Phi<\mathrm{w}_{\mathrm{x}}<\Phi_{\mathrm{p}}$, where $\delta \Phi=2.5 \mathrm{eV}<<\Phi_{\mathrm{p}}$, significantly increase their energy of motion parallel to the walls. This energy becomes the source for the upcoming SCL stage.

\section{Conclusions}

The plasma potential, the wall electron flux, and the electron temperatures calculated making use of these formulas agree well with the values obtained in particle-in-cell simulations. The SEE effect on power losses in a thruster discharge is shown to be quite different from what was predicted by previous fluid and kinetic studies. Kinetic calculation gives the values of the electron flux of a few orders of magnitude smaller than the values obtained using the fluid approach. The difference is attributed to the presence of a large depleted loss cone in the electron velocity distribution function. The EVDF in the loss cone is determined by elastic scattering of electrons due to collisions with atoms and Coulomb collisions. Our results suggest that even in the presence of a strong SEE from the walls, a contribution of the wall energy losses to the electron energy balance is much smaller than predicted by fluid theories and is proportional to the elastic scattering of electrons on collisions with atoms and ions and not inversely proportional to the electron time of flight to the walls, as is commonly assumed. It means that the wall flux is proportional to the gas density and is independent on the channel width (as long as $\mathrm{H}<<\lambda_{\mathrm{c}}$ ). This is very different from plasmas with the isotropic electron EVDF, including Maxwellian and non-Maxwellian EVDFs.

Another important result of these kinetic studies is that the SEE contribution to the current balance at the walls is self-canceled and, therefore, the plasma potential with respect to the wall and the electron energy losses on the walls are almost insensitive to the SEE. Secondary electrons emitted from the walls form two counter-streaming beams. The effective coefficient for penetration of the SEE beams from one wall to the opposite wall is equal to unity. One may assume the complete penetration of the emitted electrons because the beam electrons, which lose energy due to the two-stream instability and cannot leave the plasma in one pass between the channel walls, will eventually gain energy and escape the plasma. The SEE beams may carry a considerable portion of the cross-field electron current due to their cycloid trajectory in ExB field. This effect should depend on SEE properties of the channel wall material.

The results of these theoretical studies may explain the influence of wall material on the thruster operation and plasma parameters observed in experiments ${ }^{1,2,18}$ as well as the influence of the channel width on the electron temperature $^{22}$ by the enhancement of the electron conductivity due to contribution of the SEE electrons, rather than the enhancement of the energy losses to the walls. This conclusion is in qualitative agreement with the analysis of experimental data in Ref. 17. Future studies should be focused on generalization of this model to the twodimensional geometry.

The sheath near the electron-emitting surface may become unstable if it is characterized by the negative electron conductivity. In this paper, the negative conductivity effects in Hall thrusters are studied making use of a onedimensional particle-in-cell code. In case of intense turbulent heating, a new regime with relaxation sheath oscillations is observed. In this regime, the plasma switches between a state with non-space charge limited emission and a state with space charge limited emission. Transition to the latter state occurs when the negative conductivity appears due to modification of the velocity distribution of plasma bulk electrons. 


\section{Acknowledgments}

Simulations were carried out using the Westgrid facilities in the University of British Columbia. This research was partially supported by the Air Force Office of Scientific Research through the AF STTR Program and the U. S. Department of Energy Office of Fusion Energy Sciences.

\section{References}

\footnotetext{
1 Y. Raitses, J. Ashkenazy G. Appelbaum and M. Guelman, Proceedings of the $25^{\text {th }}$ International Electric Propulsion Conference, Cleveland, OH, August 1997, Electric Rocket Propulsion Society, Cleveland, OH, 1997 IEPC paper No.97-056.

${ }^{2}$ S. Barral, K. Makowski, Z. Peradzy'nski, N. Gaskon, and M. Dudeck, Phys. Plasmas 10, 4137 (2003).

${ }^{3}$ E. Ahedo and D. Escobar, J. Appl. Phys. 96, 983 (2004).

${ }^{4}$ G. D. Hobbs and J. A. Wesson, Plasma Phys. 9, 85 (1967).

${ }^{5}$ V.A. Rozhansky and L.D. Tsendin, Transport phenomena in partially ionized plasma (London ; New York : Taylor \& Francis 2001).

${ }^{6}$ E. Ahedo, J. M. Gallardo and M. Martinez-Sanchez, Phys. Plasmas 10, 3397 (2003).

${ }^{7}$ M. Keidar, I. Boyd and I. I. Beilis, Phys. Plasmas 8, 5315 (2001).

${ }^{8}$ N. Meeazan and M. Cappelli, Phys. Rev. E 66, 036401 (2002).

${ }^{9}$ O. Batishchev and M. Martinez-Sanchez, Proceedings of the 28th International Electric Propulsion Conference, Toulouse, France, March 2003, Electric Rocket Propulsion Society, Cleveland, OH, 2003, IEPC Paper No. 2003-188.

${ }^{10}$ D. Y. Sydorenko, and A. I. Smolyakov, Bull. Am. Phys. Soc. 261 (2004).

${ }^{11}$ D. Sydorenko, A. Smolyakov, I. Kaganovich, and Y. Raitses, Phys. Plasmas 13, 014501 (2006).

${ }^{12}$ D. Sydorenko, A. Smolyakov, I. Kaganovich, Y. Raitses, IEEE Trans. Plasma Sci. 34, 815 (2006).

${ }^{13}$ D. Sydorenko, A. Smolyakov, I. Kaganovich, Y. Raitses, Phys. Plasmas 14, 013508 (2007).

${ }^{14}$ I. Kaganovich, Y. Raitses, D. Sydorenko, A. Smolyakov, Phys. Plasmas 14, 057104 (2007).

${ }^{15}$ L.D. Tsendin, Sov. Phys. JETP 39, 805 (1974).

${ }^{16}$ I. Kaganovich, M. Misina, S. V. Berezhnoi, and R. Gijbels, Phys. Rev. E 61, 1875 (2000).

${ }^{17}$ Y. Raitses, D. Staack, A. Smirnov, and N. J. Fisch, Phys. Plasmas 12, 073507 (2005).

${ }^{18}$ Y. Raitses, D. Staack, A. Smirnov, and N. J. Fisch, Phys. Plasmas 13, 014502 (2006).

${ }^{19}$ E. Ahedo, and F. I. Parra, Phys. Plasmas 12, 073503 (2005).

${ }^{20}$ I.D. Kaganovich and L.D. Tsendin, IEEE Trans. Plasma Sci. 20, 66 (1992).

${ }^{21}$ D. Sydorenko, A. Smolyakov, I. Kaganovich, Y. Raitses, Phys. Plasmas 15, 053506 (2008).

${ }^{22}$ Y. Raitses, D. Staack, M. Keidar, and N. J. Fisch, Phys. Plasmas 12, 057104 (2005).

23 D. Sydorenko, Ph.D. thesis, University of Saskatchewan, 2006, http://library2.usask.ca/theses/available/etd-06142006111353.
} 